\title{
A niche play for stem cells
}

\section{By Lev Osherovich, Senior Writer}

Aggressive hematologic malignancies like acute lymphoblastic leukemia can blight the bone marrow and severely hamper the growth of healthy blood cells. Indeed, following chemotherapy, even the presence of a few leukemic cells can prevent bone marrow transplants from taking hold, thus impairing resumption of normal hematopoiesis.

A paper by a University of Chicago team now shows how leukemic cells impair normal hematopoiesis by reprogramming the hematopoietic stem (HS) cell niche, a specialized region at the edge of the bone marrow. The researchers suggest that inhibiting a tumor-secreted protein that lures HS cells toward these diseased niches could help in bone marrow transplantation and recovery from cancer. ${ }^{1}$

HS cells in HS cell niches ordinarily proliferate and differentiate to produce myeloid and

\section{"If we can block} recruitment of HS cells to tumor niches, we could lessen cytopenia."

-Dorothy Sipkins, University of Chicago

\section{Hematopoietic license}

Lead author Dorothy Sipkins said the work stemmed from her earlier studies on the migration of metastatic leukemia cells to specific areas of the bone marrow. ${ }^{3}$ In that study, Sipkins found that cancer cells clustered in distinct regions at the border between the bone marrow and blood vessels.

"There are very specific microenvironments in the bone marrow that promote the growth of metastatic cells," said Sipkins, who is an assistant professor of hematology and oncology at the University of Chicago.

In the new study, her team used a mouse xenograft model of ALL to show that tumor-ridden bone marrow is a magnet for HS cells. In leukemic mice injected with human HS cells, the stem cells clustered together with the cancer cells in abnormal HS cell niches. In contrast, HS cells in healthy mice were normally distributed around the bone marrow.

"When we transplanted normal cells into these tumor-infested niches, over time they migrated straight into the tumor bed," said Sipkins.

Sipkins' team found that HS cells homing to abnormal HS cell niches failed to proliferate and did not respond to G-CSF mobilization. This aberrant behavior may explain the leukocyte deficiency or cytopenia observed in ALL patients.

"Leukemic patients can have shortfalls of normal leukocytes," she said. One potential lymphoid precursor cells that mature deeper within the marrow. Upon bone injury or chemotherapy, HS cells can migrate to the spleen or to injured niches in other bones. ${ }^{2}$

In cancer patients scheduled to undergo chemotherapy, HS cells are often harvested to reintroduce them back into the patient after the cancer cells have been wiped out. However, the mobilization and recovery can be inefficient and must be boosted by therapeutics such as Mozobil plerixafor from Genzyme Corp. or two drugs from Amgen Inc.-Neupogen filgrastim and second-generation molecule Neulasta pegfilgrastim.

But for unknown reasons, not all patients respond to Neupogen or Mozobil. Several companies are thus pursuing alternative HS cell mobilization strategies and are paying attention to the new findings.

Neupogen recombinant G-CSF (CSF3) is marketed to treat neutropenia and for HS cell mobilization after chemotherapy. Mozobil, a small molecule antagonist of CXC chemokine receptor 4 (CXCR4), was approved in December for mobilization of HS cells for autologous transplantation in patients with non-Hodgkin's lymphoma (NHL) and multiple myeloma (MM).

The new study, reported in Science, suggests that cancer cells deposit a potent attractant called stem cell factor (KITLG; c-Kit ligand; SCF) in the bone marrow, leaving a false trail for HS cells and preventing their mobilization. Thus, inhibiting bone marrow SCF or raising circulating levels of SCF could divert HS cells away from entrapment in cancerous niches.

reason, Sipkins added, is because of "derangement in hematopoiesis if there's tumor infiltration into the marrow."

\section{Mechanism of inaction}

Sipkins' team next tackled the molecular mechanisms by which cancer cells recruit HS cells into abnormal bone marrow niches. The team found that Mozobil did not prevent HS cell recruitment to tumor sites, ruling out its target, CXCR4, as a player in the process.

Instead, Sipkins hypothesized that tumor cells draw HS cells to abnormal niches by secreting SCF. Her group found that SCF levels in tumor-ridden bone marrow were higher than those seen in healthy controls. They found similar increases in SCF in bone marrow from ALL and AML patients compared with healthy controls.

Indeed, leukemic mice treated with an SCF-neutralizing antibody were partially protected from the harmful effects of leukemic bone marrow, showing higher overall levels of HS cells in bone marrow and in circulation after mobilization than those seen in mock-treated controls.

Sipkins' findings thus point to bone marrow SCF as a possible therapeutic target.

"In cases where we can't mobilize with G-CSF or AMD3100 [Mozobil], targeting SCF might be a way to mobilize HS cells for autologous transfer," said Sipkins. "If we can block recruitment of HS cells to tumor niches, we could lessen cytopenia."

Ching-Cheng Chen, a postdoctoral fellow at the Institute of Stem 
Cells and Regenerative Medicine at Stanford University, cautioned that human HS cells may not behave the same way in the mouse model as they do in human bone marrow.

Chen suggested examining the effect of SCF on mobilization and homing in a recently reported model of bone marrow niche formation. ${ }^{4}$ In that study, which was published last December in Nature, Chen led a team that discovered a type of embryonic stem cell that develops into a complete bone marrow HS cell niche and could be used to seed bone marrow regrowth in damaged bone regions.

Sipkins' next step is to examine the role of SCF in solid tumors, in which she suspects a similar HS cell-subverting mechanism may be at work. Meanwhile, she has filed a patent application for targeting SCF as a therapeutic adjuvant in lymphatic cancers. The technology is available for licensing from the University of Chicago.

\section{Niche players}

SCF itself is already in the clinic to help the first step of bone marrow transplantation by drawing HS cells out of the bones and into the blood. A recombinant form of SCF called Stemgen ancestim is marketed as an adjuvant to Neupogen-mediated mobilization prior to autologous peripheral blood progenitor cell transplantation. Biovitrum AB acquired Stemgen from Amgen in December. Both companies declined to comment on the University of Chicago study.

However, the Science paper suggests that inhibiting bone marrow SCF could improve both mobilization and post-transplant engraftment. Although there are currently no SCF inhibitors in development, at least one company, SuperGen Inc., has a compound that potentially could target SCF's receptor, the proto-oncogene c-stem cell factor receptor tyrosine kinase (KIT; c-Kit; CD117).

SuperGen's MP-470 small molecule c-Kit inhibitor is in Phase Ib trials for solid tumors, and the company plans to move to Phase II this year. The company has not tested MP-470 in leukemia.

The Chicago study supports "a growing body of evidence that cancers, in particular leukemic cells, have a lot of interactions with the nonmalignant portion of the bone marrow," said SuperGen CSO David Bearss.

"We usually go after the tumor cells, but here the cancer is helped by interactions between transformed and nontransformed cells," said Bearss, who thinks it would be worthwhile to test the effect of inhibiting c-Kit in Sipkins' HS cell niche model.

c-Kit is already a target for a range of cancers, but "the idea that inhibiting c-Kit could improve mobilization is something nobody has ever thought of," he said. "Using a single targeted agent to go after the supporting environment is an elusive goal, but this study gives us an approach."

c-Kit inhibitors thus could have two therapeutic effects for the price of one: blocking tumor growth while giving HS cells a leg up in repopulating the bone marrow.

An alternative to targeting SCF or c-Kit might be a cell-based adjuvant that helps keep HS cells on track to repopulating bone marrow, said Elmar Burchardt, VP of medical affairs at Aastrom Biosciences Inc.

Burchardt suggested that the migration of purified HS cells to leukemic niches supports Aastrom's view that the behavior of transplanted stem cells is strongly influenced by the environment and can thus be modulated by formulations that are mixtures of various cell types.

Aastrom's Cardiac Repair Cell and Vascular Repair Cell therapeutics are in Phase IIb trials for cardiac myopathy and critical limb ischemia with peripheral arterial disease (PAD), respectively. The company wants to partner a third formulation, Bone Repair Cells, before starting a Phase II trial in idiopathic osteonecrosis of the femur.

Additionally, Fate Therapeutics Inc. is developing FT1050, a small molecule for ex vivo treatment of cord blood to enhance HS cell engraftment. FT1050 will enter Phase 1b trials in 1H09 for an undisclosed leukemia indication.

Osherovich, L. SciBX 2(3); doi:10.1038/scibx.2009.80

Published online Jan. 22, 2009

\section{REFERENCES}

1. Colomne, A. et al. Science; published online Dec. 19, 2008; doi:10.1126/science.1164390

Contact: Dorothy Sipkins, University of Chicago, Chicago, III. e-mail: dsipkins@medicine.bsd.uchicago.edu

2. Wilson, A. \& Trumpp, A. Nat. Rev. Immunol. 6, 93-106 (2006)

3. Sipkins, D.A. et al. Nature 435, 969-973 (2005)

4. Chan, K.F.C. et al. Nature; published online Dec. 10, 2008; doi:10.1038/nature07547

Contact: Ching-Cheng Chen, Stanford University, Stanford, Calif. e-mail: c3chen@stanford.edu

Contact: Charles K. F. Chan, same affiliation as above e-mail: chazchan@stanford.edu

COMPANIES AND INSTITUTIONS MENTIONED

Aastrom Biosciences Inc. (NASDAQ:ASTM), Ann Arbor, Mich. Amgen Inc. (NASDAQ:AMGN), Thousand Oaks, Calif. Biovitrum AB (SSE:BVT), Stockholm, Sweden

Fate Therapeutics Inc., Seattle, Wash.

Genzyme Corp. (NASDAQ:GENZ), Cambridge, Mass.

Stanford University, Stanford, Calif.

SuperGen Inc. (NASDAQ:SUPG), Dublin, Calif.

University of Chicago, Chicago, III. 